\title{
Synthesis of $\mathrm{ZnO}$ nanowires and impacts of their orientation and defects on their gas sensing properties
}

\author{
Sergio Roso, ${ }^{\mathrm{a}, \mathrm{c}}$ Frank Güell, ${ }^{\mathrm{b}}$ Paulina R. Martínez-Alanis, ${ }^{\mathrm{b}}$ Atsushi Urakawa ${ }^{\mathrm{c}}$ and Eduard Llobet ${ }^{\mathrm{a}}$ \\ ${ }^{a}$ Minos-Emas, Universitat Rovira i Virgili, Av. Països Catalans 26, 43007, Tarragona, Spain. \\ ${ }^{\mathrm{b}}$ Departament d'Electrònica, Universitat de Barcelona, c/Martí Franquès 1, 08028, Barcelona, Spain.
}

${ }^{\mathrm{c}}$ Institute of Chemical Research of Catalonia (ICIQ), The Barcelona Institute of Science and Technology, Av. Països Catalans 16, 43007, Tarragona, Spain.

$\mathrm{ZnO}$ nanowires (NWs) oriented in three different directions were synthesized via the vapour-liquid-solid technique over c-, r- and a-plane sapphire substrates. Gas sensing properties of these samples against reducing and oxidizing gases were examined. $\mathrm{ZnO} \mathrm{NWs}$ grown on the c-plane substrate showed the highest response to oxidizing gases $\left(\mathrm{NO}_{2}\right)$, while those grown on the a-plane substrate were more responsive to reducing one (ethanol). According to the insights gained by photoluminescence studies, the distinct responses were clearly correlated with the quantity and type of defects present in the material, and ultimately, relating the orientation of the different $\mathrm{ZnO} \mathrm{NWs}$ sensors to their gas sensing characteristics, showing a possible rational strategy to tailor oxide nanomaterials for gas sensing applications.

\section{Introduction}

Semiconductor metal oxides have become a major technological drive in the field of gas sensing because of their low cost, high sensitivity, fast response and their relative simplicity. Among others, nanostructured zinc oxide $(\mathrm{ZnO})^{1}$ is an interesting material for its unique electric and optoelectronic properties. Its wide direct bandgap (around $3.37 \mathrm{eV}$ ) at room temperature as well as its extremely large binding energy (around 60 meV), much higher than those of widely used metal oxides such as $\mathrm{GaN}(25 \mathrm{meV})$, make it suitable not only for gas sensing ${ }^{2}$ but also for solar cells ${ }^{3},{ }^{4}$, lasers ${ }^{5}$ and waveguides $^{6}$. Moreover, it possesses high breakdown fields, large electron saturation rates, high resistance and efficient luminescence as well ${ }^{7}$.

A variety of methodologies, such as thermal evaporation ${ }^{8}$, and hydrothermal methods ${ }^{9}$, have been reported for the synthesis of $\mathrm{ZnO}$ nanostructured in one-dimension such as nanowires (NWs). A recent report presented the use of randomly oriented $\mathrm{ZnO} \mathrm{NWs}$ as gas sensors for $\mathrm{NO}_{2}$, ethanol $(\mathrm{EtOH})$, and $\mathrm{H}_{2}$ among others ${ }^{10}$. Also, EtOH sensing properties of randomly oriented $\mathrm{ZnO}$ over silicon substrates have been investigated at different temperatures and concentrations and the high response was attributed to the high electron donating effect of EtOH compared to that of other gases like $\mathrm{CO}^{11}$. 
Regarding the photoluminescence spectra ( $\mathrm{PL}$ spectra) of $\mathrm{ZnO}$, there are different emission bands which are located in the ultraviolet and the visible (green, yellow and red/orange bands) regions. The first one is typically attributed to the characteristic emission of $\mathrm{ZnO}^{12}$, whereas the visible part is normally attributed to a variety of donor defects such as oxygen vacancies, zinc interstitials and zinc vacancies ${ }^{13}$.

In this work, the relationship between the type and quantity of defects present in the material and the gas sensing characteristics was investigated by means of photoluminescence (PL). More precisely, the aim of this paper is to study the effects of the orientation of the $\mathrm{ZnO} \mathrm{NWs}$ grown over sapphire substrates and their detailed nanostructures on the gas sensing properties.

\section{Experimental}

$\mathrm{ZnO}$ NWs were synthesized via the vapour-liquid-solid (VLS) process using a chemical vapor deposition (CVD) furnace ${ }^{14}$. It consists of three main steps: 1) Creation of the liquid alloy of the Au catalyst and the material to deposit; 2) nucleation of the material at the liquid-solid interface; and 3) growth of the NWs. The nucleation and growth of the solid $\mathrm{ZnO} \mathrm{NWs}$ occur due to supersaturation of the liquid droplet. A schematic view of the VLS process can be observed in Fig 1. Previously to the growth of the metal oxide NWs, a $3 \mathrm{~nm}$ Au thin layer that will act as a catalyst was deposited by sputtering onto the sapphire substrates. The precursor was a powder of $\mathrm{ZnO}$ and graphite (125 $\mathrm{mg}$ ), and Ar was used as carrier gas. Both the precursor and the substrate were placed on a quartz boat into the horizontal CVD furnace. The substrate was placed $1 \mathrm{~cm}$ away from the precursor and samples were grown in different batches. Then, the temperature was raised to $900^{\circ} \mathrm{C}$ and kept constant for 30 minutes. The optimum Ar gas flow was $400 \mathrm{ml} / \mathrm{min}$. To synthesize $\mathrm{ZnO} \mathrm{NWs}$ via VLS, it is essential to decompose the $\mathrm{ZnO}$ powder, and to enable this decomposition to occur at $850^{\circ} \mathrm{C}$, the $\mathrm{ZnO}$ powder is mixed with graphite. All the process was carried out under atmospheric pressure. It goes without saying that the relative humidity has no influence on the growth of the $\mathrm{ZnO}$ NWs. Moreover, the temperature at which the growth takes place $\left(900^{\circ} \mathrm{C}\right)$ the relative humidity present can be neglected. 


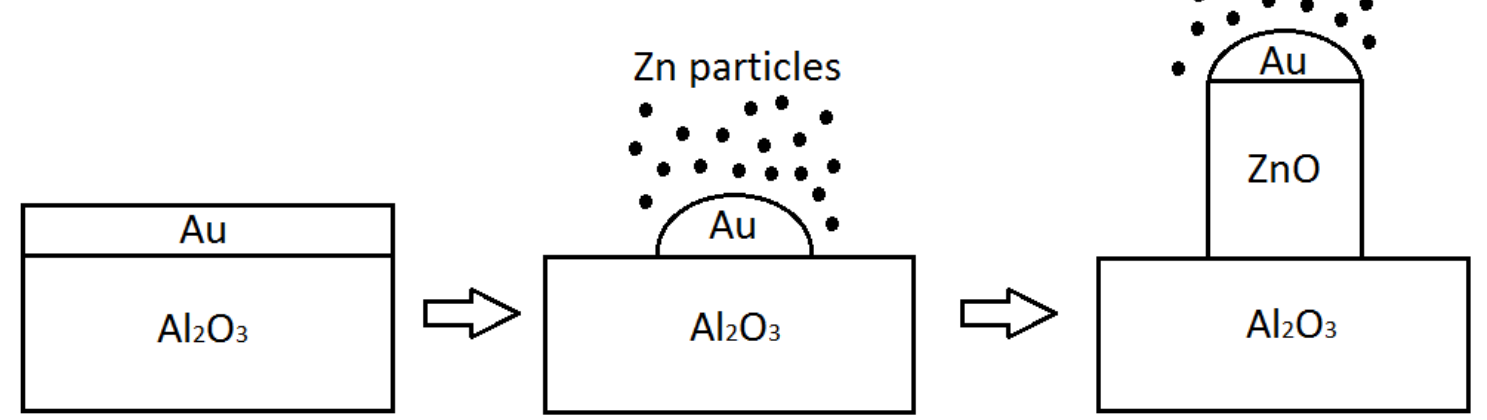

Fig. 1: Schematic view of the VLS process. First, at high temperatures, the Au thin layer turns into droplets. Then, $\mathrm{Zn}$ atoms condense and attach to the Au droplet. Finally, when the Au droplet becomes supersaturated, the bottom-up growth of the $\mathrm{ZnO} \mathrm{NW}$ takes place.

Three different types of sapphire $\left(\mathrm{Al}_{2} \mathrm{O}_{3}\right)$ substrates were used, with different crystallographic directions in order to grow $\mathrm{ZnO}$ NWs oriented along the c-plane (0001), r-plane (1-102) and a-plane (11-20). The match between the lattice parameter of the $\mathrm{ZnO}$ and that of the sapphire will allow us to epitaxially grow the $\mathrm{ZnO}$ NWs. The structural characterization was carried out by a field-emission scanning electron microscopy (FESEM, Hitachi H-4100F) and by X-ray diffraction (XRD, Bruker-AXS D8-Discover diffractometer). For obtaining the PL spectra, a He-Cd laser at $325 \mathrm{~nm}$ was used in order to overcome the band gap barrier of the ZnO NWs. The luminescence was dispersed by an Oriel Instruments 74000 monochromator and detected with a Hamamatsu H8259-02 with a socket assembly E717-500 photomultiplier. All sources of noise were removed with a Stanford Research System SR830 DSP lock-in amplifier. In order to measure the resistance of our sensors, silver (Heraeus, AD1688-06) parallel electrodes were deposited on top of the active layer. Substrates were glued to a hotplate in order to measure at different operating temperatures (i.e. sensors could be operated from room temperature up to $250^{\circ} \mathrm{C}$ ). Gas mixing and delivery were performed employing a computer controlled mass-flow system.

\section{Results and Discussion}

FESEM images of the ZnO NWs grown over the three sapphire substrates (c-, r-, and aplanes) are presented in Fig. 2. In all cases, the diameter of the NWs was about $70 \mathrm{~nm}$ and the length was about $1 \mu \mathrm{m}$. The dimension of the NWs was dependent on the deposition time and also the thickness of the Au layer, being deposited prior to the synthesis and working as a catalyst during the $\mathrm{ZnO} N W$ growth ${ }^{15}$. Hence, these two 
parameters were kept the same for the three samples. As evident from Fig. 2, distinct orientations of the $\mathrm{ZnO}$ NWs were observed for the different sapphire substrates used.
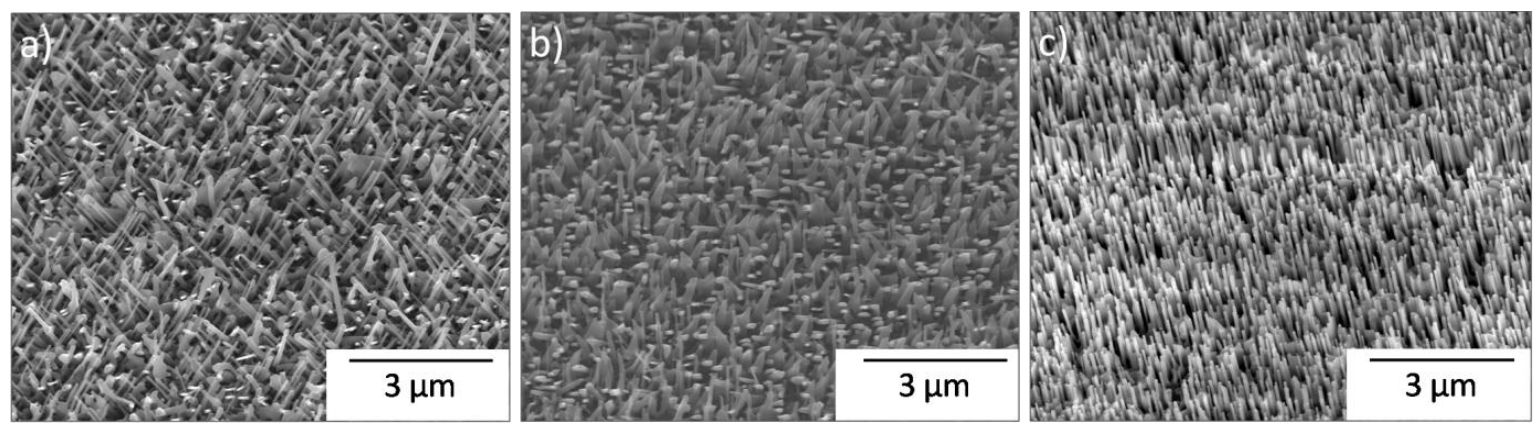

Fig. 2: SEM images of the $\mathrm{ZnO} N W$ s grown over a) c-, b) r-, and c) a-planes of sapphire

For the c-plane substrate (Fig. 2a), the NWs grew tilted at 51 and $129^{\circ}$, relative to the substrate. For the r-plane (Fig. 2b), the NWs showed a conical shape; they did not exhibit a well-defined orientation and many wire-to-wire junctions were present. This could be explained by the generation of stress during the initial stage of growth, thus joining together to make thicker structures along the growth ${ }^{15}$. Finally, for the a-plane (Fig. 2c), the NWs grew vertically aligned. It is well known that NWs grow following the exposed crystalline structure of the substrate.

Nucleation at the initial stage has a crucial role in in-plane alignment of the NWs. It is known that $\mathrm{ZnO}$ nuclei grow with an epitaxial relationship with, in this case, c-, a- and r-plane sapphire due to the lattice match between them ${ }^{16}$. The c- a- and r-planes of sapphire and the c-plane of $\mathrm{ZnO}$ are related by a factor of 4 , with a mismatch of less than $0.08 \%$ at room temperature. This relationship leads to vertical epitaxial growth on $\mathrm{ZnO} \mathrm{NWs}$ on top of a-plane sapphire substrates ${ }^{17}$. The reduced lattice mismatch between $\mathrm{ZnO}$ and sapphire is generally attributed to homogenous (isotropic) orientations ${ }^{18}$. The visual inspection shows that the lattice matching is more significant between $\mathrm{ZnO} \mathrm{NW}$ and c- and a-plane sapphire substrates as evidenced by strong preferential orientation of the NW growth directions.

XRD measurements were performed and the patterns showed $\mathrm{ZnO}$ NWs with a hexagonal crystalline structure (Fig. 3) in accordance with ICDD card 00-036-1451. The major peaks can be indexed to the (002) as well as the (101) among other crystal planes. The intensities of the two peaks differ drastically, indicating strong preferential orientation of $\mathrm{ZnO} \mathrm{NWs}$ grown along specific crystal plane(s). Notably, the 101 
reflection was fully diminished for NWs grown over the r-plane. This implies preferable orientation in the $<001>$ direction of NWs. Also, it should be noticed that the peak at $33^{\circ}$ in the r-plane XRD pattern (see Fig. 3 ) is originated from the substrate.

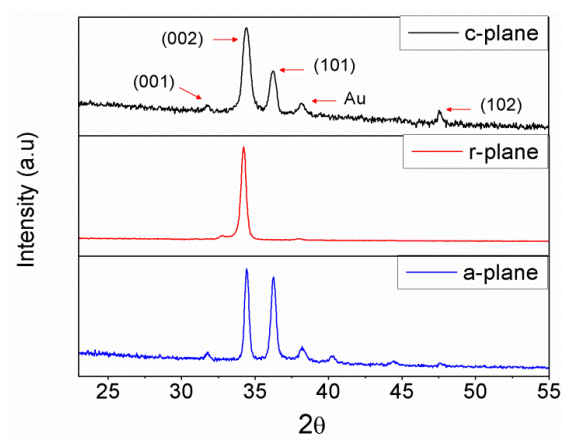

Fig. 3: XRD patterns of ZnO NWs grown on c-, r- and a-plane sapphire substrates.

Furthermore, these three $\mathrm{ZnO}$ NWs were subject to gas sensing tests using an oxidizing gas $\left(\mathrm{NO}_{2}\right)$ and reducing vapour $(\mathrm{EtOH})$ by means of DC resistance measurements at different operating temperatures $\left(150-250^{\circ} \mathrm{C}\right)$. All sensors were exposed to $30 \mathrm{~min}$ of a given concentration of a species, followed by a 60 min cleaning phase in dry air. All sensors experienced an increase or decrease in resistance under exposure to oxidising or reducing gases, respectively. This implies that $\mathrm{ZnO}$ behaves as an n-type semiconductor. Fig. 4 shows representative response/recovery cycles for the three sensors operated at $250^{\circ} \mathrm{C}$ against ethanol and nitrogen dioxide. The intensity of the response increased with gas concentration. Responses were reproducible in all cases. The baseline resistance could be fully recovered when cleaning in air after a sufficiently long time. The response time for all sensors is about 5 minutes and, even though the recovery time is longer, these times are still is in the same range than those reported for nanowire gas sensors ${ }^{19,20}$. Although every sensor response does not reach complete stabilization (r-plane and c-plane), it is observed that the a-plane sensor does reach such stabilization. Taking this sensor as a reference the response and recover times have been calculated as the time that the sensor takes to achieve $90 \%$ of the total value of the response.

Fig. 5 shows the dependence of sensor response as a function of the operating temperature at a fixed concentration of EtOH $(500 \mathrm{ppm})$ and $\mathrm{NO}_{2}(100 \mathrm{ppm})$. All three sensors showed the highest response when operated at $250^{\circ} \mathrm{C}$. Interestingly, for EtOH, the highest response was achieved by the a-plane sensor, whereas for $\mathrm{NO}_{2}$ the highest response was that of the c-plane sensor. 
Mechanistically, at first oxygen is physisorbed on the surface of the $\mathrm{ZnO} \mathrm{NW}$. Then, it becomes chemisorbed by attracting electrons of the conduction band, which forms a depletion region on the surface of the $\mathrm{ZnO}$ NWs and consequently a potential barrier between each nanowire, resulting in an increase in the resistance. It has been reported that EtOH can undergo various reactions such as dehydration and dehydrogenation. However, since $\mathrm{ZnO}$ is a basic oxide, dehydrogenation is favoured. In this case, the ethanol sensing mechanism is controlled by the change (i.e. lowering) in the equilibrium concentration of chemisorbed, negatively charged oxygen species, which results in a change in the width of the depletion region at the surface of the $\mathrm{NWs}^{21}$.

In the case of $\mathrm{NO}_{2}$ detection, either the equilibrium concentration of chemisorbed oxygen species is raised or $\mathrm{NO}_{2}$ is directly chemisorbed on the surface of the n-type $\mathrm{ZnO} \mathrm{NWs}^{22}$. These oxidizing molecules extract free electrons from the conduction band of $\mathrm{ZnO}$ and thus, the width of the depletion region is further extended, which increases the resistance of NWs.
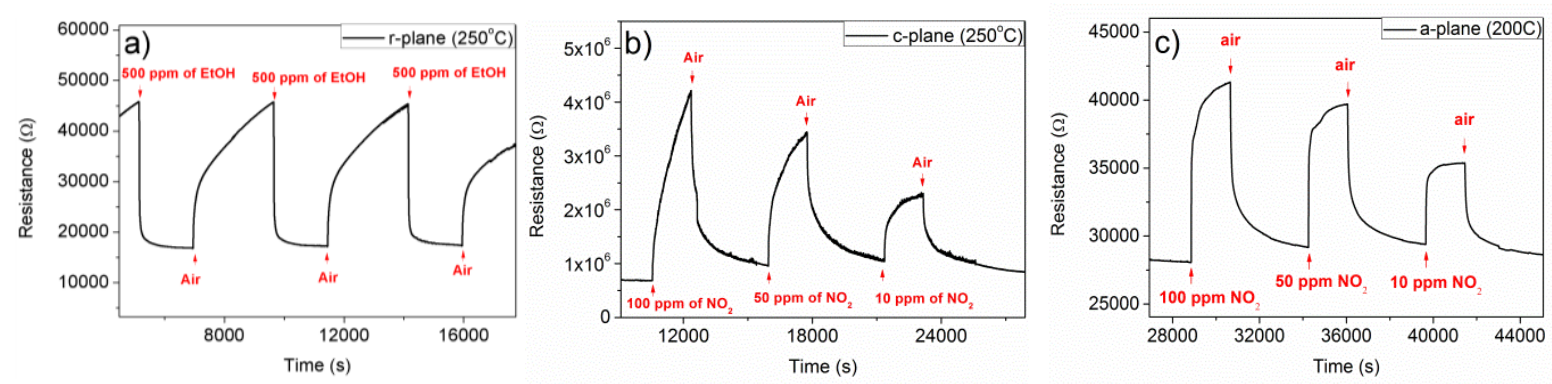

Fig. 4: Responses of the three $\mathrm{ZnO} \mathrm{NW}$ sensors to different gases: a) EtOH, b) and c) $\mathrm{NO}_{2}$.

According to the results presented in Fig. 5, the c-plane sensor presented the best response to $\mathrm{NO}_{2}$ while the a-plane sensor exhibited the best response to $\mathrm{EtOH}$ vapours. In chemo-resistive sensors, there are many factors that influence sensor response. In addition to the morphology of the active surface structure, the presence of defects and their types can influence the electronic properties of materials and thus gas sensing properties. As a matter of fact, it is accepted that the overall response of gas sensors is a contribution both from the morphology of the material and the crystalline properties of the material itself ${ }^{23}$. Due to our set-up, we cannot increase the temperature further than $250^{\circ} \mathrm{C}$. Most likely at higher temperatures (i.e. $>300^{\circ} \mathrm{C}$ ) the response will decrease. The reason behind such behaviour could be that the oxygen would not chemisorb on the active sites anymore and thus, the response of the sensor will dramatically decrease ${ }^{24}$. 

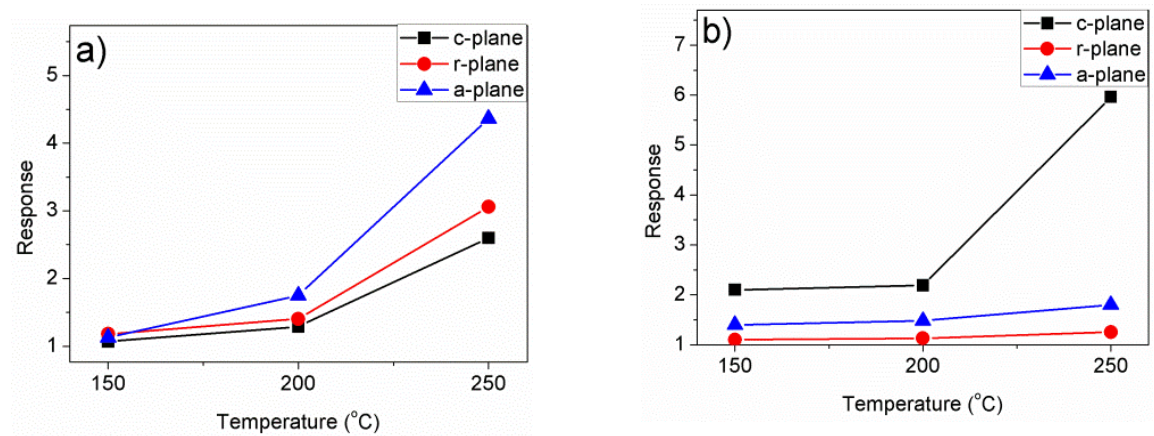

Fig. 5: Temperature-dependent responses of the three $\mathrm{ZnO} \mathrm{NW}$ sensors to a) EtOH and b) $\mathrm{NO}_{2}$. Response was defined as $\mathrm{R}_{\mathrm{ai}} \mathrm{r} / \mathrm{R}_{\mathrm{EtOH}}$ and $\mathrm{R}_{\mathrm{NO} 2} / \mathrm{R}_{\mathrm{air}}$, respectively.

In order to verify this statement for these $\mathrm{ZnO}$ NW sensors, PL experiments were performed at room temperature (Fig. 6). Two different zones in the spectra can be clearly identified. The first one, which corresponds to the high peak located at the lower wavelength (around $380 \mathrm{~nm}$ ), is associated to the near-band edge emission of the material. This strong emission corresponds to the recombination of electrons from the minimum of the conduction band with holes of the valence band of the semiconducting $\mathrm{ZnO}^{25,26}$. Additionally, by zooming in the visible light region of the spectra (see the inset in Fig. 6), the presence of an emission band that ranges from 440 to nearly $820 \mathrm{~nm}$ is revealed, related to the large surface-to-volume ratio of the $\mathrm{ZnO} \mathrm{NWs}$. The exact origin of such a broad emission band has remained controversial; nevertheless it has been generally attributed to defects and impurities referred to as a deep-level, trap-state or impurity-related radiative recombination process (such as oxygen vacancies, Zn interstitials, oxide antisite defect ${ }^{27}$, and zinc vacancies). Therefore, the low intensity of this visible emission indicates the high crystalline quality of the different samples grown (i.e. NWs have a low density of defects).

The spectra also show a shift in the position of the maximum of the visible light emission. For the $\mathrm{ZnO}$ NWs grown over the c-plane the maximum is located at $550 \mathrm{~nm}$ (i.e., yellow luminescent band), while for those grown over a- and r-planes, the maximum is located near 520nm (i.e., green luminescent band).

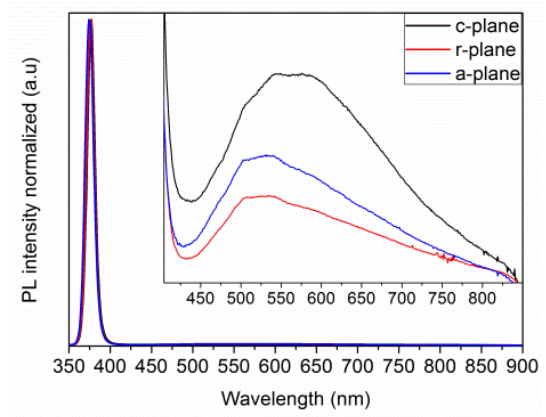


Fig. 6: Room-temperature PL spectra of the three $\mathrm{ZnO}$ NW sample.

Typically, defects responsible for the yellow luminescent band (YL) have been excess of oxygen and the presence of oxygen interstitials (Oi). Nevertheless, for the green luminescent band (GL), it has been reported to be originated from oxygen vacancies (Vo). However, the presence of both GL and YL in the same samples suggests a common origin for them ${ }^{28}$. We will assume that the formation of such defects occurs during the growth process. Thereby, the initial Vo will form at the surface. Then, as Zn and $\mathrm{O}$ atoms are supplied, by adsorption, the $\mathrm{ZnO}$ layer will be created. By deposition of more atoms, the created defect will be overgrown and will become a Vo inside the lattice $^{29}$. Consequently, if the orientation is different, the Vo will form from a different kind of surface.

If we correlate now the PL spectra to the gas response to $\mathrm{NO}_{2}$, the c-plane sensor, i.e. the one that shows the highest response to $\mathrm{NO}_{2}$, employs the material with the most intense visible emission band in its PL spectrum, centred at 550, which corresponds to the YL. This means that the sensor with the maximum number of oxygen interstitials is the most sensitive to $\mathrm{NO}_{2}$. Sensor response to $\mathrm{NO}_{2}$ can be explained as follows; $\mathrm{NO}_{2}$ molecules adsorb on the material surface. Then, this adsorbed molecule acts as an acceptor according to the following reactions ${ }^{30}$ :

$$
\begin{aligned}
& \mathrm{NO}_{2} \text { (gas) } \rightarrow \mathrm{NO}_{2} \text { (ads) } \\
& e^{-}+\mathrm{NO}_{2}(\text { ads }) \rightarrow \mathrm{NO}_{2}^{-}(\text {ads })
\end{aligned}
$$

Furthermore, the tilted morphology of $\mathrm{ZnO} \mathrm{NWs}$ in the c-plane sensor favours the presence of many nanowire to nanowire junctions, the space charge regions of which are modulated upon $\mathrm{NO}_{2}$ chemisorption. This further enhances the response towards nitrogen dioxide of the c-plane sensor as shown in Fig. 5 b.

However, this does not seem to be the case for EtOH sensing. Fig. 5(a) shows that the most sensitive sensor for EtOH sensing is the a-plane sensor. In this particular case, as the maximum in the PL spectra corresponds to the GL, the origin for this visible band is related to oxygen vacancies. As mentioned above, oxygen vacancies are most likely responsible for the YL band. These vacant points would act as active sites in which oxygen will chemisorb during gas sensing ${ }^{31}$. The more numerous the active sites, the more oxygen will be chemisorbed and consequently, more molecules of target gas (EtOH in this case) will react and the sensor response will be dramatically affected. 
This suggests that the engineering of defects in single crystalline metal oxides could be a viable approach to ameliorate selectivity.

\section{Conclusions}

To sum up, it has been shown that the morphology and orientation of $\mathrm{ZnO}$ NWs, CVDgrown on sapphire substrates are highly dependent on the crystalline plane of the substrate. By employing room-temperature PL studies, it has been possible to establish that the crystalline plane of the substrate has also an impact in the number of defects and in the nature of these (i.e., defects located at the surface or deep-levels within the $\mathrm{ZnO}$ NWs). Sensors have been fabricated and tested employing $\mathrm{ZnO}$ NWs with different orientations. For the detection of nitrogen dioxide, it was found that the response of $\mathrm{ZnO}$ NWs was directly correlated to the overall amount of defects. The higher the number of defects is (c-plane), the higher the response to nitrogen dioxide is. On the other hand, for the detection of ethanol, $\mathrm{ZnO} \mathrm{NWs}$ with an intermediate number of defects (i.e. a-plane) in which surface defects were dominant led to the best results. These significant differences revealed by this study suggest that engineering the amount and nature of defects in metal oxide NWs deserves further research, since it may become an effective strategy for enhancing and tuning the selectivity of metal oxide sensors upon demands.

\section{Acknowledgements}

This work has been funded in part by MINECO under grant no. TEC2012-32420. S. Roso and A. Urakawa would like to thank MINECO for support through Severo Ochoa Excellence Accreditation 2014-2018 (SEV -2013-0319). S. Roso is also grateful to the URV-ICIQ fellowship. F. Güell and P.R. Martínez-Alanis acknowledge the financial support from CONACyT through grants no. 203519 and 232459, respectively.

\section{Notes and References}

1. J. M. Wu, Y.-R. Chen and Y.-H. Lin, Nanoscale, 2011, 3, 1053-1058.

2. M. R. Alenezi, S. J. Henley, N. G. Emerson and S. R. P. Silva, Nanoscale, 2014, 6, 235247.

3. M. Law, L. E. Greene, J. C. Johnson, R. Saykally and P. Yang, Nat Mater, 2005, 4, 455459. 
4. J. Fan, Y. Hao, C. Munuera, M. García-Hernández, F. Güell, E. M. J. Johansson, G. Boschloo, A. Hagfeldt and A. Cabot, The Journal of Physical Chemistry C, 2013, 117, 16349-16356.

5. D. Vanmaekelbergh and L. K. van Vugt, Nanoscale, 2011, 3, 2783-2800.

6. S. Chu, G. Wang, W. Zhou, Y. Lin, L. Chernyak, J. Zhao, J. Kong, L. Li, J. Ren and J. Liu, Nat Nano, 2011, 6, 506-510.

7. J. Cui, Materials Characterization, 2012, 64, 43-52.

8. H. V. Han, N. V. Hieu and T. Trung, Science of Advanced Materials, 2014, 6, 1659-1667.

9. H. Jang, B. Son, H. Song, G. Jung and H. Ko, J Mater Sci, 2014, 49, 8000-8009.

10. H. Nguyen, C. T. Quy, N. D. Hoa, N. T. Lam, N. V. Duy, V. V. Quang and N. V. Hieu, Sensors and Actuators B: Chemical, 2014, 193, 888-894.

11. P. Rai, R. Khan, R. Ahmad, Y.-B. Hahn, I.-H. Lee and Y.-T. Yu, Current Applied Physics, 2013, 13, 1769-1773.

12. J. Smith, A. Akbari-Sharbaf, M. J. Ward, M. W. Murphy, G. Fanchini and T. Kong Sham, Journal of Applied Physics, 2013, 113, 093104.

13. H. Zeng, G. Duan, Y. Li, S. Yang, X. Xu and W. Cai, Advanced Functional Materials, 2010, 20, 561-572.

14. F. Güell, J. O. Ossó, A. R. Goñi, A. Cornet and J. R. Morante, Superlattices and Microstructures, 2009, 45, 271-276.

15. C. Weigand, J. Tveit, C. Ladam, R. Holmestad, J. Grepstad and H. Weman, Journal of Crystal Growth, 2012, 355, 52-58.

16. W. I. Park, D. H. Kim, S.-W. Jung and G.-C. Yi, Applied Physics Letters, 2002, 80, $4232-$ 4234.

17. M. H. Huang, S. Mao, H. Feick, H. Yan, Y. Wu, H. Kind, E. Weber, R. Russo and P. Yang, Science, 2001, 292, 1897-1899.

18. N. Han, P. Hu, A. Zuo, D. Zhang, Y. Tian and Y. Chen, Sensors and Actuators B: Chemical, 2010, 145, 114-119.

19. F. Hernandez-Ramirez, J. D. Prades, A. Tarancon, S. Barth, O. Casals, R. Jimenez-Diaz, E. Pellicer, J. Rodriguez, J. R. Morante, M. A. Juli, S. Mathur and A. Romano-Rodriguez, Advanced Functional Materials, 2008, 18, 2990-2994.

20. J. D. Prades, R. Jimenez-Diaz, F. Hernandez-Ramirez, S. Barth, A. Cirera, A. RomanoRodriguez, S. Mathur and J. R. Morante, Applied Physics Letters, 2008, 93, 123110.

21. J. Xu, J. Han, Y. Zhang, Y. a. Sun and B. Xie, Sensors and Actuators B: Chemical, 2008, 132, 334-339.

22. M.-W. Ahn, K.-S. Park, J.-H. Heo, J.-G. Park, D.-W. Kim, K. J. Choi, J.-H. Lee and S.-H. Hong, Applied Physics Letters, 2008, 93, 263103.

23. C. Navío, S. Vallejos, T. Stoycheva, E. Llobet, X. Correig, R. Snyders, C. Blackman, P. Umek, X. Ke, G. Van Tendeloo and C. Bittencourt, Materials Chemistry and Physics, 2012, 134, 809-813.

24. E. R. Waclawik, J. Chang, A. Ponzoni, I. Concina, D. Zappa, E. Comini, N. Motta, G. Faglia and G. Sberveglieri, Beilstein Journal of Nanotechnology, 2012, 3, 368-377.

25. J. S. Reparaz, F. Güell, M. R. Wagner, A. Hoffmann, A. Cornet and J. R. Morante, Applied Physics Letters, 2010, 96, -.

26. R. Aad, V. Simic, L. Le Cunff, L. Rocha, V. Sallet, C. Sartel, A. Lusson, C. Couteau and G. Lerondel, Nanoscale, 2013, 5, 9176-9180.

27. B. Lin, Z. Fu and Y. Jia, Applied Physics Letters, 2001, 79, 943-945.

28. O. Marin, G. Grinblat, A. M. Gennaro, M. Tirado, R. R. Koropecki and D. Comedi, Superlattices and Microstructures, 2015, 79, 29-37.

29. K. Subannajui, C. Wongchoosuk, N. Ramgir, C. Wang, Y. Yang, A. Hartel, V. Cimalla and M. Zacharias, Journal of Applied Physics, 2012, 112, 034311.

30. S. Öztürk, N. Kılınç and Z. Z. Öztürk, Journal of Alloys and Compounds, 2013, 581, 196201. 
31. S. Pati, S. B. Majumder and P. Banerji, Journal of Alloys and Compounds, 2012, 541, 376-379. 